\title{
Performance Management bei der Polizei - wie Führungskräfte Informationssysteme nutzen
}

\author{
Rolf Ritsert/Michael Evers
}

Der Beitrag analysiert wie Führungskräfte von vier Polizeien Führungsinformationen nutzen und welche Faktoren das Nutzungsverhalten beeinflussen. Viele Polizeien haben ihre Führungs- und Organisationssysteme im Sinne eines Performance Managements ergebnis- und wirkungsorientiert ausgerichtet und komplexe Führungsinformationssysteme aufgebaut. Der Beitrag fasst die Ergebnisse von fünf Studien mit quantitativer und/oder qualitativer Methodik zusammen. Im Ergebnis zeigt sich, dass die Führungskräfte die Informationen primär zur Wissenserweiterung, nachrangig zur Entscheidungsdurchsetzung und weniger zur Entscheidungsfindung nutzen. Die Akzeptanz der Anwender wird als bedeutendster Faktor für das Nutzungsverhalten identifiziert. Für die Faktoren Behördengröße, Verhalten der Behördenleitung und Datenqualität lassen sich ebenfalls Effekte nachweisen. Die Ergebnisse unterstreichen die Notwendigkeit, Konzepte nicht isoliert, sondern umfassend unter Berücksichtigung von Kultur und Strategie der Organisation einzuführen. Zudem ergeben sich daraus grundlegende Empfehlungen für die Einführung und Konzeption von Führungsinformationssystemen.

\section{Performance Management und Führungsinformationssysteme bei der Polizei}

Viele Public-Management-Reformansätze der letzten Jahrzehnte haben den Fokus auf das Performance Management, im Sinne einer systematischen, leistungs- und wirkungsorientierten Steuerung, gelenkt. Die Ausrichtung auf die Wirksamkeit und

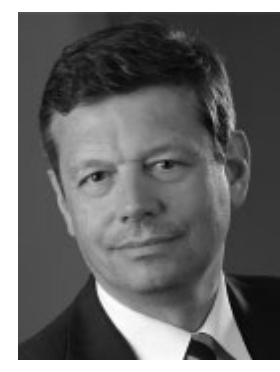

Prof. Dr. Rolf Ritsert

Universitätsprofessor und Leiter des Fachgebietes Betriebswirtschaftslehre - Public Management an der Deutschen Hochschule der Polizei in Münster.
Ergebnisse des Verwaltungshandelns kann mittels Strategien, Zielvereinbarungsverfahren und Budgets erfolgen. Diese Form der Verwaltungssteuerung erzeugt einen hohen Bedarf an Informationen in Form von Kennzahlen, so genannter Performance-Indikatoren, die häufig in Führungsinformationssystemen zusammengefasst werden.

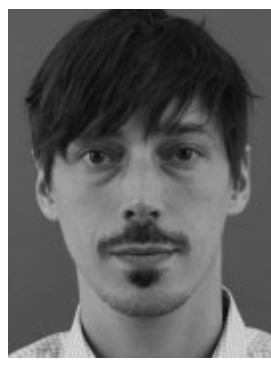

Michael Evers, M.A. Wissenschaftlicher Mitarbeiter des Fachgebietes Betriebswirtschaftslehre - Public Management an der Deutschen Hochschule der Polizei in Münster.
Seit Mitte der 1990er-Jahre haben die Neuen Steuerungsmodelle die Polizeien der Länder und des Bundes in sehr unterschiedlicher Art und Weise beeinflusst. Erfolgreiche Weiterentwicklungen der Führungs- und Organisationsysteme stehen ernüchternden Erfahrungen gegenüber - das Thema Management bleibt daher bei den Polizeien ambivalent. Ungeachtet dessen sind Controlling- und Zielvereinbarungsverfahren bei den Polizeien häufig implementiert und werden von den Verantwortlichen überwiegend als nützlich bewertet. ${ }^{1}$ Damit einhergehend entstanden bei vielen Polizeien Führungsinformationssysteme, die unterschiedliche Daten zusammenfassen, z.B. die Polizeiliche Kriminalstatistik (PKS), Verkehrsunfalldaten, Daten aus den polizeilichen Vorgangsbearbeitungsprogrammen und Einsatzbearbeitungssystemen, das Haushaltsmanagementsystem, allgemeine statistische Daten (z.B. Einwohnerzahlen, Kfz-Zulassungszahlen usw.) und Daten besonderer polizeilicher Tätigkeiten (z.B. Maßnahmen der Spurensicherungen, die Anzahl von Kfz-Kontrollen o.ä.). Die Verarbeitung der umfangreichen Datenmengen erfolgt teilweise mit Standard-Softwareanwendungen, vereinzelt werden auch komplexe Data-Warehouse-Systeme genutzt, z.B. das Führungsinformationssystem der Polizei Baden-Württemberg. ${ }^{2}$

Befürworter betonen die Möglichkeiten einer sachbezogenen Entscheidungsfindung bis hin zu einer umfassenden Rati-

Vgl. Ritsert et al. 2016, S. 29.

Vgl. Renter 2017, S. $877 \mathrm{ff}$. 


\begin{tabular}{|c|c|c|c|c|c|}
\hline & $\begin{array}{l}\text { Hattwig/ } \\
\text { Ritsert (2011) }\end{array}$ & $\begin{array}{l}\text { Hohenlohe } \\
\text { (2012) }\end{array}$ & \begin{tabular}{|l} 
Nierenz/ \\
Ritsert (2013)
\end{tabular} & $\begin{array}{l}\text { Waschulewski } \\
(2017)\end{array}$ & $\begin{array}{l}\text { Lehmann/ } \\
\text { Evers (2019) }\end{array}$ \\
\hline Polizei (Land) & $\begin{array}{l}\text { Nordrhein- } \\
\text { Westfalen }\end{array}$ & Sachsen & $\begin{array}{l}\text { Nordrhein- } \\
\text { Westfalen }\end{array}$ & $\begin{array}{l}\text { Mecklenburg-Vor- } \\
\text { pommern }\end{array}$ & $\begin{array}{l}\text { Baden- } \\
\text { Württemberg }\end{array}$ \\
\hline Stichprobe & $\begin{array}{l}17 \text { Führungskräfte } \\
\text { einer ausgewählten } \\
\text { Großbehörde in NRW }\end{array}$ & $\begin{array}{l}44 \text { Revierleiter der } \\
\text { sächsischen Polizei }\end{array}$ & $\begin{array}{l}176 \text { Führungskräfte } \\
\text { der KPB in NRW }\end{array}$ & $\begin{array}{l}8 \text { Polizeiinspektions- } \\
\text { leiter der Polizei M-V }\end{array}$ & $\begin{array}{l}101 \text { Mitarbeiter/Sach- } \\
\text { bearbeiter, } \\
\text { 3 Führungskräfte }\end{array}$ \\
\hline Methode & $\begin{array}{l}\text { qualitative Inter- } \\
\text { views, quantitative } \\
\text { Befragung } \\
\end{array}$ & $\begin{array}{l}\text { quantitative (Tele- } \\
\text { fon-) Befragung }\end{array}$ & $\begin{array}{l}\text { quantitative (Online-) } \\
\text { Befragung }\end{array}$ & qualitative Interviews & $\begin{array}{l}\text { Qualitative Inter- } \\
\text { views, quantitative } \\
\text { (Online-) Befragung } \\
\end{array}$ \\
\hline $\begin{array}{l}\text { Datenerhe- } \\
\text { bung }\end{array}$ & Juni 2009 & April 2012 & Mai 2012 & Mai-Juni 2017 & März-Juli 2018 \\
\hline $\begin{array}{l}\text { betrachtete } \\
\text { Systeme }\end{array}$ & $\begin{array}{l}\text { FISPol NRW (Schnitt- } \\
\text { stellen zu u.a. PKS, } \\
\text { Verkehrsunfalldatei, } \\
\text { PVP, e-Cebius) }\end{array}$ & $\begin{array}{l}\text { PASS, IVO, EMS-Web, } \\
\text { DPNA, PKS, EUSka, } \\
\text { Vollzugspolizeiliche } \\
\text { Statistik, FVS }\end{array}$ & $\begin{array}{l}\text { FISPol NRW (Schnitt- } \\
\text { stellen zu u.a. PKS, } \\
\text { Verkehrsunfalldatei, } \\
\text { PVP, e-Cebius) }\end{array}$ & $\begin{array}{l}\text { PIA, Standard- } \\
\text { berichte, PKS }\end{array}$ & FIS BW, BSC, POLAS \\
\hline
\end{tabular}

Tab 1: Studien zur Nutzung von Führungsinformationen

onalitätssicherung der Führung. Kritiker wenden dagegen ein, dass die aus der Privatwirtschaft entlehnten Konzepte zwar im Außenverhältnis eine Legitimität erzeugten, im Innenverhältnis aber mit der Verwaltungskultur nicht immer vereinbar seien. Infolgedessen zeigten sich Entkopplungseffekte der propagierten Steuerungsverfahren mit dem tatsächlichen Verwaltungshandeln. Es handele sich demzufolge nicht um eine Rationalitätssicherung, sondern eher um einen Rationalitätsmythos. Daher kommt der Frage, ob und inwiefern Führungskräfte die Informationssysteme tatsächlich nutzen, eine besondere Bedeutung zu.

\section{Studien zur Nutzung von Führungsinformationen}

Die Tabelle 1 zeigt einen Überblick von fünf Studien zur Nutzung von Führungsinformationen bei den Polizeien. Die Studien untersuchen Polizeien in vier Bundesländern mit unterschiedlichen Führungsinformationssystemen. In Nordrhein-Westfalen (Führungsinformationssystem der Polizei NRW - FISPol NRW) und Baden-Württemberg (Führungsinformationssystem - FIS BW) nutzen die Polizeien integrierte Führungsinformationssysteme. FISPol NRW verfügt z.B. über Schnittstellen zu polizeiinternen Datensystemen, wie zur PKS, zur Verkehrsunfalldatei, zum Vorgangsbearbeitungssystem Polizei-Vorgangsprogramm (PVP), oder zum Einsatzbearbeitungssystem e-Cebius. Das strategische Steuerungsinstrument Balanced Scorecard (BSC) und das Poli- zeiauskunftssystem (POLAS) sind u.a. Elemente des FIS BW. In Mecklenburg-Vorpommern erfolgt eine software-gestützte Datenauswertung mittels der Anwendung Polizeiliche Information und Auswertung (PIA), die so genannte Standardberichte erzeugt. In Sachsen stehen den Führungskräften unterschiedliche Systeme zur Verfügung, z.B. das Auskunftssystem Sachsen (PASS), das Integrierte Vorgangsbearbeitungssystem (IVO), das Einsatzmanagement Sachsen (EMS-Web), das IT-Verfahren Dienstplanung, Dienstzeitnachweis und Dienstzeitabrechnung (DPNA) sowie weitere Informationen der PKS, der Elektronischen Unfalltypenstreckkarte (EUSka), der Vollzugspolizeilichen Statistik, oder des Fortbildungsverwaltungssystems (FVS).

Die Befragten waren Führungskräfte, die grundsätzlich dem höheren Polizeivollzugsdienst angehören. Die Datenerhebung erfolgte qualitativ und quantitativ mittels Face-to-face-Interviews sowie Online- oder Telefonbefragungen. Die Studien betrachteten das Nutzungsverhalten, hier verstanden als die unterschiedlichen Nutzungsarten, die Nutzungshäufigkeit und die Nutzungsintensität sowie teilweise deren Einflussfaktoren.

\section{Informationsnutzung zur Entscheidungsfindung (instru- mentelle Nutzung)}

Die Nutzung von Informationen lässt sich in drei Arten differenzieren, nämlich die instrumentelle, die symbolische und die konzeptionelle Nutzung. Die instrumentelle Nutzung bezeichnet eine direkte Verwendung der Kennzahlen zur Entscheidungsfindung, also zur Willensbildung. Dabei werden verschiedene Entscheidungsmöglichkeiten abgewogen und diejenige gewählt, die aufgrund der Informations- und Kennzahlenanalyse als am besten geeignet erscheint. Diese Form der sachbezogenen Entscheidungsfindung wird häufig im Zuge von PerformanceManagement-Ansätzen propagiert, so dass man einen hohen Anteil der instrumentellen Nutzung hätte erwarten können. Korrespondierend bestätigten einige Befragte, dass sie die Führungsinformationen zur Entscheidungsfindung nutzen, dieses als sehr bedeutsam ansehen und Aspekte erkannten, die sie zuvor nicht in die Entscheidung einbezogen hätten: ${ }^{3}$

„[Controlling-Informationen spielen bei der Entscheidungsfindung] eine erhebliche Rolle! Ein Großteil aller Entscheidungen, die gefällt werden, beziehen sich auf Controlling-Informationen..."

„Man hat relativ wenig, worauf man Führungsentscheidungen fußen lassen kann. Controlling-Informationen 
sind daher eine Basis für Entscheidungen.“

„Ich hätte das Problem, dass ich [ohne Controlling-Informationen] gar nicht zur Entscheidung käme, weil ich das Problem nicht vor Augen hätte.“

Die Befragten erläuterten, dass thematische Schwerpunktsetzungen und damit einhergehende, konkrete Personalentscheidungen (z.B. belastungsorientierte Festlegung der Schichtstärken, o.ä.) ohne fundierte Daten nur mit einem Verlust der Entscheidungsqualität möglich seien. Trotzdem bestätigte sich die Annahme nicht, dass die instrumentelle Nutzung die
Die Führungsinformationen helfen z.B. dabei, Personalentscheidungen und interne Aufgabenverschiebungen gegenüber Nachgeordneten zu begründen bzw. Steuerungsentscheidungen mit Bezug auf die Einsatzbelastung nachvollziehbarer darzulegen:

„Wenn ein Revierleiter sagt, ich kann das nicht leisten, und ich nehme ein vergleichbares Revier und rufe dann die Zahlen auf und sage, warum kann es denn da funktionieren, bei noch einer schlechteren Personaldecke? “

Gegenüber übergeordneten Instanzen hatten Entscheidungen im Hinblick auf Ressourcenzuweisungen einen besonders hohen Stellenwert für die Führungskräfte.

\section{"Letztendlich werden Entscheidungen durchgesetzt, indem sie mit den Daten aus den Informationssystemen transparent gemacht werden."}

am häufigsten angewandte Art der Informationsnutzung sei. Im Gegenteil, in den Studien Nierenz/Ritsert 2013 und Hohenlohe 2012 zeigte sich, dass die instrumentelle Nutzung am geringsten ausgeprägt ist.

\section{Informationsnutzung zur Ent- scheidungsdurchsetzung (symbo- lische Nutzung)}

Die symbolische Nutzung setzt bei der Willensdurchsetzung an. Führungskräfte nutzen Informationen symbolisch, wenn sie andere Akteure beeinflussen wollen, um bereits getroffene Entscheidungen zu legitimieren oder durchzusetzen. In den Interviews gaben die Führungskräfte an, dass die Transparenz und Nachvollziehbarkeit von Entscheidungen der Schlüssel zu deren Durchsetzung seien:

„...Letztendlich setze ich meine Entscheidungen durch, indem ich sie transparent mache.“
Mitunter entstand der Eindruck, als wäre es die vorrangige Funktion der Informationen, Ressourcenforderungen durchzusetzen:

„...Gerade in Ressourcenfragen werden Sie nur über das Visualisieren und Erklären von Controlling-Daten zu Akzeptanz kommen. Da reichen keine guten Worte aus, sondern da muss man ganz knallhart sagen: ,So ist die Situation, da entwickeln wir uns hin, wenn wir nicht gegensteuern'. Dazu sind Controlling-Daten existenziell wichtig!“

„...Das sind dann Controlling-Daten, [die zur Durchsetzung von Entscheidungen genutzt werden,] wenn es darum geht, Ressourcen zugewiesen zu bekommen. Beispiel: Kräftezuweisung anhand der Einsatzbelastung. “

Dazu passt auch der Befund der Studie Hohenlohe 2012, dass Daten zur Dienstplanung, zum Einsatzmanagement und zur Einsatzbelastung häufiger genutzt werden, als z.B. die PKS oder Verkehrsunfallstatistiken.

Die Befragten kritisierten teilweise eine „Scheinrationalität“. Die Zahlen würden selektiv so zusammengestellt, um die Ressourcenforderungen der jeweiligen Akteure zu begründen und stünden damit einer einheitlichen rationalen Bewertung entgegen:

„...Wenn man es schafft, eine zentra-

le Stelle zu schaffen, die objektiv mit Controlling-Daten umgeht und keine Eigeninteressen hat, dann kann man zum Teil auch diese sachfremde Kontrolle als emotionale Lage dahinter ausschalten und man hat den großen Vorteil, dass man nicht immer wieder zu unterschiedlichen Ergebnissen kommt..."

Die Befunde sind bedeutsam, da sie auf theoretisch motivierte Zweifel des Performance-Management-Ansatzes hindeuten, insbesondere im Hinblick auf die Annahme, dass die Organisationen die Führungsinformationssysteme nutzen, um nach Effektivität zu streben. Die Erkenntnisse weisen auf Bereichsegoismen im Sinne des methodischen Individualismus hin. Demnach strebten die Organisationsmitglieder eher nach Eigeninteressen und Ressourcenmehrung als nach der Effektivität der Organisation. Zudem könnten diese Befunde auch darauf hinweisen, dass die Organisationen im Sinne des Resource-Dependence-Ansatzes zuerst danach streben, ihre Unsicherheitszonen im Hinblick auf Ressourcen zu kontrollieren, bevor sie ihre Effektivität zu steigern versuchen.

\section{Wissen und verstehen (konzeptio- nelle Nutzung)}

In den Studien Nierenz/Ritsert 2013 und Hohenlohe 2012 zeigte sich, dass die konzeptionelle Nutzung bei den Befragten am stärksten ausgeprägt ist. Konzeptionelle Nutzung bedeutet, dass Nutzer die Wissensbasis und das Verständnis des Aufgabenfeldes unabhängig von konkreten Entscheidungen erweitern. Sie ist also nicht unmittelbar an ein konkretes Problem oder eine aktuelle Entscheidungssituation gebunden. Das erworbene Wissen oder Aufgabenverständnis dient dazu, zukünftige Entwicklungen besser einzuschätzen 
und kann ggf. zu einem späteren Zeitpunkt hilfreich bei der Entscheidungsfindung oder -durchsetzung sein:

„Das ist für mich eigentlich auch einer der großen Mehrwerte von den zur Verfügung gestellten Tabellen. Dass man sehen kann, wer löst das Problem scheinbar besser als andere. Und dass man das eben als Initiative, als Anpacker nimmt, um nachzuforschen."

„Wenn ich aber Organisation darauf ausrichten will mit entsprechenden Personal-, Sach- und Finanzmitteln [...], muss ich irgendwann konkrete Informationen haben, wie sich der Bereich entwickelt, sonst kann ich als Führungskraft organisatorisch die Vorsorge dafür gar nicht treffen, dass dieser Bereich künftig arbeitsfähig ist."

Konkret gaben die Befragten an, dass sie die Führungsinformationen konzeptionell u.a. bei Abweichungskontrollen bzw. SollIst-Vergleichen (z.B. Kriminalitätslage, Verkehrsunfallbekämpfung), der Abschätzung von Entwicklungen (z.B. Personalplanung und Ressourcenbedarf), Dienststellenvergleichen, oder der Analyse der Vorgangs- und Einsatzbelastung nutzen. ${ }^{4}$

\section{Der bedeutendste Einflussfaktor - die Akzeptanz der Anwender}

Die Frage, warum Führungskräfte Informationen nutzen und welche Faktoren das Nutzungsverhalten beeinflussen, bleibt eine bedeutende Fragestellung des Performance Management. Das Nutzungsverhalten kann von persönlichen Faktoren der Nutzer (z.B. Einstellung, Alter, Wissen, Bedürfnisse im Hinblick auf extrinsische Belohnungen), organisatorischen Faktoren (Alter, Größe, Aufgabe, sowie Ziel- und Ergebnisorientierung der Organisation), den Informationssystemen (z.B. Verfügbarkeit der Informationen und der Datenqualität) oder weiteren externen Faktoren (Einfluss und Erwartungen externer Stakeholder, z.B. politische Kontrolle, Bürgerinteresse usw.) abhängen. ${ }^{5}$

Nierenz/Ritsert 2013 untersuchten sechs relevante Einflussfaktoren im Hinblick auf das Nutzungsverhalten, nämlich das Wissen des Nutzers, die wahrgenom-

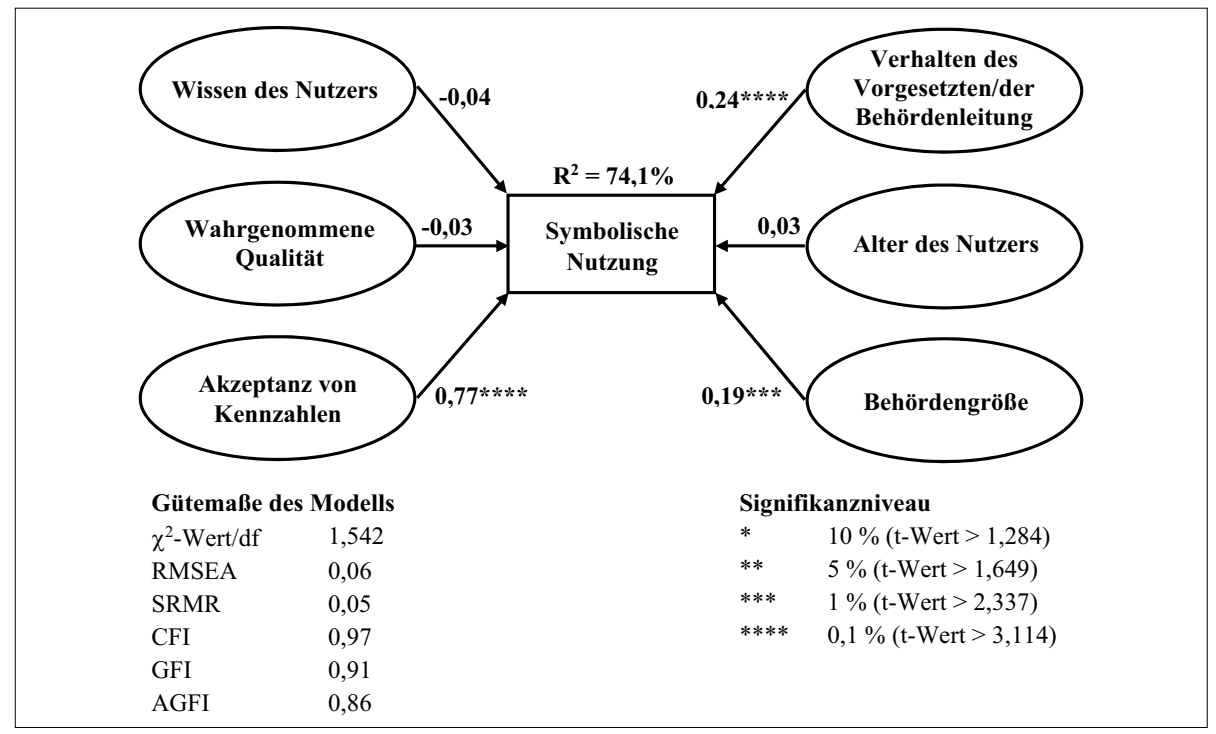

Abb. 1: Einflussfaktoren auf die symbolische Nutzung (Vgl. Nierenz/Ritsert 2013, S. 160)

mene Datenqualität, die Akzeptanz des Nutzers von Kennzahlen, das Verhalten des Vorgesetzten/der Behördenleitung, das Alter des Nutzers sowie die Behördengröße. Das Nutzungsverhalten wurde differenziert betrachtet - zum einen nach den drei Nutzungsarten, der Nutzungshäufigkeit und der Nutzungsintensität. Im Ergebnis zeigte sich, dass nur ein Faktor einen signifikanten Einfluss auf alle untersuchten Aspekte hatte, nämlich die Akzeptanz des Nutzers im Hinblick auf Kennzahlen. Das Konstrukt der Akzeptanz von Kennzahlen wurde wie folgt operationalisiert:

1. Kennzahlen sind ein Steuerungsinstrument, das auch für die Polizei gut geeignet ist,

2. Ich habe eine positive Einstellung $\mathrm{zu}$ Kennzahlen,

3. Kennzahlen unterstützen mich wesentlich bei meiner Arbeit als Führungskraft und

4. Meine Mitarbeiter haben eine positive Einstellung zu Kennzahlen.

Die Experten unterstrichen ebenfalls häufig die besondere Bedeutung der Akzeptanz und den Nutzen der Informationssysteme für ihre Arbeit:

„Es ist nicht wegdenkbar in unserer heutigen Zeit. Das sind ganz wichtige Grundlagen, die ich benötige, um meine Inspektion vernünftig steuern zu können. Also ohne diese Daten geht es nicht."

Interessant ist auch der Befund von Hattwig/Ritsert 2011, dass die Befragten die eigene Akzeptanz als sehr hoch einstuften, jedoch meinten, dies gelte nicht für alle Führungskräfte der Behörde. Die besondere Bedeutung der Akzeptanz im Hinblick auf die nachgeordneten Mitarbeiter betonten viele Befragte:

„Es geht insbesondere darum Akzep$\operatorname{tanz}$ zu schaffen. Akzeptanz bei den Mitarbeitern für die gewählten Ziele [und Kennzahlen]. Die größte Herausforderung ist es eben diese Akzep$\operatorname{tanz}$ zu generieren.“

Die Abbildung 1 veranschaulicht, dass im Falle der symbolischen Nutzung neben der Akzeptanz auch die Einflussfaktoren Behördengröße und Verhalten des Vorgesetzten/der Behördenleitung einen signifikanten aber deutlich geringeren Einfluss auf das Nutzungsverhalten hatten. Die Abbildung zeigt auch, dass sowohl die Gütemasse des Modells als auch die Varianzen mit dem Modell hinreichend zu erklären waren $\left(\mathrm{R}^{2}=74,1 \%\right)$.

Dagegen erwies sich bei der instrumentellen und konzeptionellen Nutzung neben der Akzeptanz kein weiterer Faktor als relevant und signifikant. Damit konnte der Nachweis geführt werden, dass die Einflussfaktoren im Hinblick auf die Nut-

4 Vgl. Hattwig/Ritsert 2011, S. 89 f., Waschulewski 2017, S. $38 \mathrm{ff}$.

5 Vgl. Moynihan/Pandey 2010, S. $850 \mathrm{ff}$. 
zungsart variieren und daher bei wissenschaftlichen Analysen berücksichtigt werden sollten. Im Hinblick auf weitere nutzerbezogene Faktoren, also das Wissen, die Diensterfahrung und das Alter der Nutzer konnten keine oder nur marginale Effekte auf das Nutzungsverhalten nachgewiesen werden.

Bei der durch die Anwender wahrgenommenen Datenqualität zeigen sich uneinheitliche Befunde im Hinblick auf das Nutzungsverhalten. Die quantitative Analyse Nierenz/Ritsert 2013 zeigte keine Effekte beim Nutzungsverhalten und nur geringe Effekte bei der Nutzungshäufigkeit. Allerdings erklärt das verwendete Modell die Streuung der Variablen nicht hinreichend $\left(\mathrm{R}^{2}=19,9 \%\right)$. In qualitativen Studien ergaben sich durchaus Hinweise auf die Relevanz der Datenqualität im Sinne einer Richtigkeit, Vollständigkeit und Aktualität der Daten. Wenn die Führungskräfte auf die Richtigkeit und Validität vertrauen, erscheint eine häufigere Nutzung wahrscheinlicher:

„[...] wenn die über einen Monat veraltet sind, dann kommt der Einfluss zu spät, wenn man reagieren will. [...] Und wir wollen uns ja nicht überraschen lassen, wir wollen ja wissen, wie das aktuell wirklich aussieht.“

„Man muss auf der Grundlage der Daten auch unangenehme Entscheidungen treffen, deswegen ist es schon wichtig, dass man nicht die Diskussion über die Daten, sondern über die Ursachen der Daten führt.“

\section{Hinweise auf dysfunktionales Verhalten}

Bei den qualitativen Studien zeigen sich Hinweise auf dysfunktionales Verhalten von Mitarbeitern aus Sicht der Organisation. Vereinzelt gab es Anzeichen für unbeabsichtigte Lenkungseffekte, auch Manipulationen von Daten seien nicht immer fernliegend. Theoretisch lässt sich dieses Phänomen mittels der Goal Setting Theory erklären. Demnach kann eine wahrgenommene hohe Verpflichtung zur Erreichung von Zielen, die Organisationsmitglieder zu dysfunktionalen Verhalten veranlassen.

„Das hinterlege ich bereits in diesem

Zielvereinbarungsprozess, dass wir eben auch keine Zahlen frisieren oder Zahlen schönen, sondern dass wir in dem Zielerreichungsprozess ein Abbild unserer Tätigkeit haben wollen.“

„Wenn Mitarbeiter mir eine saubere Erklärung liefern, warum dieses oder jenes Ziel nicht erreicht werden konnte und es ist nachvollziehbar, dann kann ich das akzeptieren. Dann ist mir dieses nicht erreichte Ziel mit einer sauberen Erklärung lieber, als wenn ein Mitarbeiter mir nur die
2. Die in vielen Managementkonzepten propagierte Informationsnutzung zur Entscheidungsfindung, also die instrumentelle Nutzung, stellt die am geringsten ausgeprägte Nutzungsart dar. Die Analyse des Nutzungsverhaltens zeigt, dass die konzeptionelle Nutzung, also der Ausbau der persönlichen Wissensbasis und das Verständnis des Aufgabenfeldes, dominiert. Dieser Befund steht auch im Einklang mit empirischen Analysen bei einer Kommunalverwaltung oder privaten Unternehmen. ${ }^{7}$ Um eine konzeptionelle Nutzung

\section{"Solche Systeme sollten nicht isoliert, sondern im Einklang mit einer ziel- und ergebnisorientierten Führungs- und Organisationskultur eingeführt werden."}

\begin{abstract}
Zahlen liefert, weil er mir sie liefern muss und die Zahlen sind geschönt.“
\end{abstract}

Daher betonen viele Führungskräfte die besondere Bedeutung der Vermittlung der Philosophie, der Vision und des Zwecks der Steuerungsverfahren sowie die Etablierung einer konstruktiven Fehlerkultur. ${ }^{6}$

\section{Wesentliche Schlussfolgerungen und Empfehlungen}

1. Die Führungsinformationssysteme bei der Polizei werden weiter eine hohe Bedeutung haben. Einerseits führt die Digitalisierung dazu, dass die Möglichkeiten, relevante polizeiliche Daten zu erfassen, $\mathrm{zu}$ speichern und auszuwerten, weiter zunehmen. Andererseits führt die Zentralisierung von Polizeistrukturen auch zu höheren Führungsspannen und damit zu einer Verdichtung bei den Führungsaufgaben. Infolgedessen werden die Möglichkeiten einer direkten personalen Führung weiter zugunsten der indirekten, auf Systemen beruhenden Führung verschoben. zu unterstützen, sollte bei der Konzeption der Führungsinformationssysteme auf eine hohe Ausgewogenheit der Kennzahlen geachtet werden. Eine solche ganzheitliche Betrachtung des polizeilichen Aufgabenfeldes umfasst insbesondere Aspekte der Führung, der Mitarbeiter, der Kooperationspartner und der Finanzen. Hier besteht grundsätzlich Verbesserungspotenzial, da beispielsweise bei den Polizeien kaum Führungsinformationssysteme existieren, die die Finanzfunktion mit allen Haushaltsdaten vollständig integriert haben.

3. Die Akzeptanz der Nutzer, hier verstanden als die persönliche Einstellung sowie die wahrgenommene Einstellung der Mitarbeiter zu den Führungssystemen konnte als bedeutendster Faktor für die Nutzung identifiziert werden. Dieser Befund unterstreicht die Bedeutung, solche

6 Vgl. Renter 2017, S. $877 \mathrm{ff}$.

7 Vgl. Hunold 2003, S. 169. 
Systeme nicht isoliert, sondern im Einklang mit einer ziel- und ergebnisorientierten Führungs- und Organisationskultur einzuführen. Auch andere Studien haben den Effekt beschrieben, dass sich die Ziel- und Ergebnisorientierung der Organisation förderlich auf die Nutzung von Performance-Informationen auswirken. ${ }^{8}$ Zudem unterstreichen weitere Studien die besondere Bedeutung des Aufbaus einer Organisationskultur, die eine Nutzung von Performance-Informationen fördert. ${ }^{9}$ Führungskräfte der Polizei unterstreichen, dass eine konstruktive Fehlerkultur eine zwingende Voraussetzung für die Etablierung von Führungsinformationssystemen sei, auch um dysfunktionales Verhalten von Mitarbeitern möglichst zu verhindern.

4. Aufgrund der Ergebnisse der qualitativen Studien erscheint ein Einfluss der Datenqualität auf das Nutzungsverhalten bedeutsam zu sein. Einen solchen Befund bestätigen auch andere Studien. ${ }^{10}$ Die uneinheitlichen Ergebnisse könnten durch die unterschiedliche technische Qualität der Systeme begründet sein. Während die Polizei Baden-Württemberg über ein leistungsfähiges Data-Warehouse-System verfügt, erfolgt anderenorts das Datenmanagement nicht automatisiert mittels der Standard-Software Excel. Ein automatisiertes und integriertes Datenmanagement erscheint eine wichtige Voraussetzung für fehlerfreie und aktuelle Daten zu sein, daher sollten grundsätzlich leistungsfähige Data-Warehouse-Systeme genutzt werden. Landuyt 2009.

$10 \mathrm{Vgl}$. Ammons/Rivenbark 2008.

\section{Literatur}

Ammons, D. N./Rivenbark W. C. (2008): Factors influencing the use of performance data to improve municipal services: Evidence from the North Carolina benchmarking project, in: Public Administration Review, 68, S. 304-318.

Broadnax, W. D./Conway, K. J. (2001): The social security administration and performance management, in: Forsythe, D. (Hrsg.): Quicker, better, cheaper: Managing performance in American government, Albany, S. 143-175.

De Lancer Julnes, P./Holzer, M. (2001): Promoting the utilization of performance measures in public organizations: An empirical study of factors affecting adoption and implementation, in: Public Administration Review, 61, S. 693-708.

Hattwig, M./Ritsert, R. (2011): Führung und Controlling - eine empirische Untersuchung am Beispiel einer nordrhein-westfälischen Kreispolizeibehörde, in: Ritsert, R./Vera, A. (Hrsg.): Polizei und Management, Schriftenreihe der Deutschen Hochschule der Polizei, Münster, S. 67-101. Hohenlohe, H. (2012): Einflussfaktoren für die Nutzung von Führungsinformationen, dargestellt am Beispiel der Polizei des Freistaates Sachsen. Deutsche Hochschule der Polizei, Münster.

Hunold, C. (2003): Erfolgsfaktoren der kommunalen Kostenrechnung, in: Weber, J./Kunz, J. (Hrsg.): Empirische Controllingforschung. Begründung - Beispiele - Ergebnisse, Wiesbaden, S. 153-177 und S. 422-431.

Lehmann, M./Evers, M. (2019): Empirische Analyse der Motivationswirkung und des Nutzungsverhaltens des Führungs- und Steuerungssystems ausgewählter Polizeireviere des Polizeipräsidiums Tuttlingen/Baden-Württemberg im Hinblick auf Führungskräfte und Mitarbeiter (im Erscheinen).

Moynihan, D. P./Landuyt, N. (2009): How do public organizations learn? Bridging structural and cultural divides, in: Public Administration Review, 69, S. 1097-1105.

Moynihan, D. P./Pandey, S. K. (2010): The Big Question for Performance Management: Why Do Managers Use Performance Information?, in: Journal of Public Administration Research and Theory, 20(4), S. 849-866.

Nierenz, C. B./Ritsert, R. (2013): Wie Führungskräfte Kennzahlen nutzen: Nutzungsverhalten und Einflussfaktoren, in: Ritsert, R./Vera, A. (Hrsg.): Von Streifenpolizisten, Spezialisten und Führungskräften: ausgewählte empirische Studien zur Optimierung der Polizeiarbeit, Schriftenreihe Polizeiwissenschaft in Theorie und Empirie, Band 3, Frankfurt/Main, S. 145-168.

Renter, R. (2017): Von der Vision zur Konzeption - Strategische Steuerung der Polizei BW mithilfe des Führungsinformationssystems und der Balanced Scorecard, in: Stierle, J./Wehe, D./ Siller, H. (Hrsg.): Handbuch Polizeimanagement, Heidelberg, S. 867-894.

Ritsert, R./Rickards, R. C./Evers, M. (2016): Management Tool Usage and Perceived Benefits: Insights from Germany's Police Forces, in: European Police Science and Research Bulletin, 14, S. 26-33.

Waschulewski, F. (2017): Wie Führungskräfte Kennzahlen nutzen: Nutzungsverhalten und Einflussfaktoren am Beispiel der Polizeiinspektionen Mecklenburg-Vorpommerns. Deutsche Hochschule der Polizei, Münster. 